\title{
Inclinación de Incisivos Maxilares en Sujetos con Deformi- dad Facial de Clase III. Opciones en Cirugía Primero
}

\author{
Maxillary Incisor Inclination in Subjects With Class III Facial Deformity. Options in Surgery First.
}

\author{
Leonardo Brito*; Javier Villa*; Pablo Navarro*; Juan Pablo Alister ${ }^{* *, * * *, * * * * * ;}$ Francisca Uribe ${ }^{* * * * * * * * * * * * *}$ \& Sergio Olate ${ }^{* * * * *, * * * * * * *}$
}

BRITO, L.; VILLA, J.; NAVARRO, P.; ALISTER, J.P.; URIBE, F.; OLATE, S. Inclinación de incisivos maxilares en sujetos don deformidad facial de clase III. Opciones en cirugía primero. Int. J. Morphol., 34(4):1502-1505, 2016

RESUMEN: El objetivo de esta investigación es determinar la inclinación de incisivos maxilares en sujetos con maloclusión de clase III. Se incluyeron 35 sujetos que presentaran deformidad facial de clase III (DF-III) para analizar la tomografía computarizada de haz cónico; las mediciones se realizaron para los cuatro incisivos maxilares identificando el plano palatino considerado entre espina nasal anterior y posterior y el eje axial dentario obtenido por la unión entre punto coronal y punto apical; los resultados mostraron angulaciones de $111,6^{\circ}$ a $113,7^{\circ}$ para los incisivos, lo cual permite obtener valores cercanos a la normalidad de angulación dentaria. Se concluye que los sujetos seleccionados presentan una angulación dentaria adecuada para la realización de procedimientos de cirugía primero.

PALABRAS CLAVE: Inclinación de incisivo; Mal oclusión clase III; Cirugía primero

\section{INTRODUCCIÓN}

La inclinación dentaria de incisivos en el sector superior e inferior tienen un rol fundamental en la articulación y función dentaria; la oclusión tiene un papel importante en las funciones estomatognaticas, de forma que la posición y angulación dentaria puede determinar la función de un sujeto (Troy et al., 2009). En situaciones clínicas con presencia de deformidades faciales existen importantes alteraciones funcionales que relacionan la oclusión con otros factores (Pereira-Stabile et al., 2012).

Las alteraciones o anomalías faciales de clase III (DFIII) se caracterizan por presentar una posición retruída de maxila y una posición adelantada de mandíbula, lo que genera graves alteraciones funcionales. Recientes investigaciones han demostrado que esta anomalía se relaciona con alteraciones nasales significativas (Olate et al., 2014; Olate et al., 2015), alteraciones en posición de sínfisis (Velásquez et al., 2015) y alteraciones en ATM (Muñoz et al., 2014a; Muñoz et al., 2014b; Goulart et al., 2015); sin duda, estas condiciones determinan la necesidad de realizar tratamien- tos quirúrgicos mediante cirugía ortognática y cirugía ortofacial, lo cual exige tradicionalmente el empleo de ortodoncia en las diferentes fases del tratamiento.

La cirugía primero ha ganado aceptación en los últimos años, transformándose en un proceso categorizado y organizado (Huang \& Chen, 2015). Su éxito depende, entre otras variables, de la posición dentaria y la angulación dentaria en la etapa postoperatoria; la cirugía primero permite disminuir las alteraciones faciales y dentales de la preparación ortodoncia pre quirúrgica y disminuir los tiempos de tratamiento (Hernandez-Alfaro \& Guijarro-Martinez, 2014), de forma que la posición dentaria en sujetos con deformidades faciales son relevantes.

El objetivo de esta investigación es determinar la angulación dentaria y definir las condiciones que permitirían la inclusión de estos sujetos bajo protocolos de cirugía primero.

\footnotetext{
Programa de Magister en Odontología, Universidad de La Frontera, Temuco, Chile.

** Programa de Doctorado en Ciencias Médicas, Universidad de La Frontera, Temuco, Chile.

**** Becario CONICYT-PCHA/Doctorado Nacional/ 2015-21150598.

***** Becario CONICYT-PCHA/Doctorado Nacional/2015-21150752.

****** División de Cirugía Oral y Maxilofacial, Universidad de La Frontera, Temuco, Chile.

******* Centro de Investigación Biomédica, Universidad Autónoma de Chile, Temuco, Chile.
} 


\section{MATERIAL Y MÉTODO}

La presente investigación consideró la aprobación del comité de bioética científica de la Universidad de la Frontera (folio $\mathrm{n}^{\circ}$ 162/15). Se incluyeron 35 sujetos consecutivos que consultaron en la División de Cirugía Oral y Maxilofacial de la Universidad de La Frontera bajo el diagnóstico de deformidad facial de clase III.

Requisitos de inclusión fueron las de presentar estudios tomográficos (tomografía computadorizada de haz cónico, CTBC) de pacientes con deformidad facial de clase III (DF-III), con una edad de entre 18 y 30, sin limitación por sexo o etnia y que accedieran libremente a la investigación. Se excluyeron sujetos que presentaran cirugía maxilofacial previa, sujetos con tratamiento ortodoncico pasado o presente, pacientes con exodoncias previas (exceptuando tercer molar) y sujetos con malformaciones maxilofaciales.

El diagnóstico de los sujetos con DF-III se realizó clínicamente, confirmando una retrusión maxilar y prognatismo mandibular y a nivel dentario identificando un overjet igual o menor a cero y una relación molar de clase III de Angle. En la imagen se realizaron técnicas cefalométricas para identificar la relación de DF-III.

Las mediciones fueron realizadas por un único operador previamente calibrado en dos ocasiones con 15 días de diferencia usando el programa EZ-plus 3D software (EWOO Technology Co, Ltd. Korea), aplicando el análisis morfométrico en el plano sagital considerando los cuatro dientes incisivos maxilares (incisivo central derecho, ICD; incisivo central izquierdo, ICI; incisivo lateral derecho, ILD; incisivo lateral izquierdo, ILI).

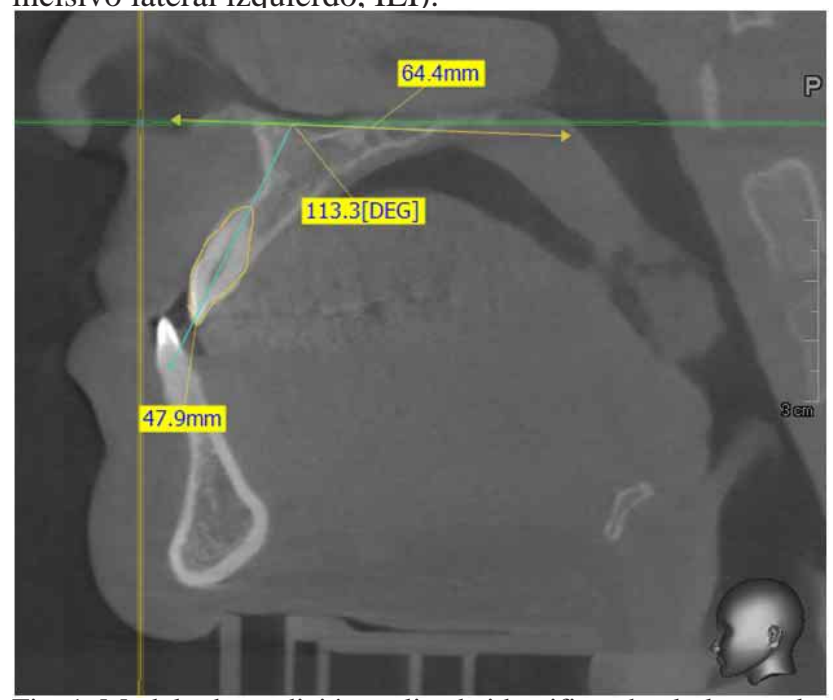

Fig. 1. Modelo de medición realizada identificando el plano palatino y el plano axial del incisivo a evaluar
Para ello se determinó el plano palatino (PP), considerado como la línea que une la espina nasal anterior (EA) y la espina nasal posterior (EP); las líneas dentarias fueron orientadas según el eje axial de cada diente uniendo el punto mas coronal con el punto mas apical (Fig. 1); cada ángulo fue registrado para determinar la condición en cada diente. Los datos obtenidos fueron registrados en una tabla Excel; se realizó estadística descriptiva y la valoración de la correlación intraclase (ICC).

\section{RESULTADOS}

Las mediciones realizadas en los diferentes dientes demostraron tener una relación positiva en la correlación intraclase considerando con valores sobre los 0,982 , determinando intervalos de confianza sobre 95\% (Tabla I).

Tabla I. Correlación intraclase en el analisismoorfometrico de dientes incisivos maxilares

\begin{tabular}{cc}
\hline Diente & ICC \\
\hline ICD & 0,985 \\
ICI & 0,982 \\
ILD & 0,994 \\
ILI & 0,992 \\
\hline
\end{tabular}

La Tabla II muestra las angulaciones obtenidas para los dientes ILD, ICD, ICI, ILI, evidenciando valores próximos entre ellos; entre incisivos centrales, existe un promedio de angulación de $113,7^{\circ}$, sin diferencias entre ellas, mientras que entre incisivos laterales existe una diferencia promedio de $1,6^{\circ}$, siendo de $111,6^{\circ}$ en el incisivo lateral derecho y de $113,2^{\circ}$ en el incisivo lateral izquierdo. La Fig. 2 muestra la distribución de angulaciones obtenidas para todos los sujetos; en general, los incisivos laterales presentan una mayor angulación que los incisivos centrales

Tabla II. angulaciones obtenidas para los diferentes dientes analizados.

\begin{tabular}{llllc}
\hline & Máximo & Mínimo & Promedio & D. E. \\
\hline LD & $126,3^{\circ}$ & $91,9^{\circ}$ & $111,6^{\circ}$ & $8.7^{\circ}$ \\
CD & $128,1^{\circ}$ & $88,9^{\circ}$ & $113,7^{\circ}$ & $8^{\circ}$ \\
CI & $129,4^{\circ}$ & $88,3^{\circ}$ & $113,7^{\circ}$ & $9,5^{\circ}$ \\
LI & $128^{\circ}$ & $95^{\circ}$ & $113,2^{\circ}$ & $8,2^{\circ}$ \\
\hline
\end{tabular}



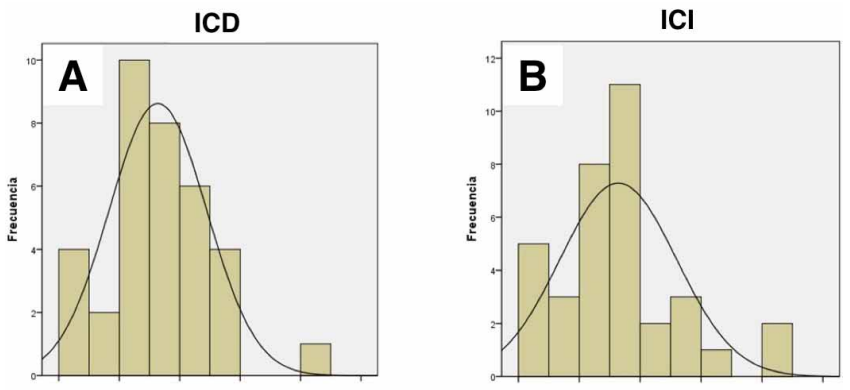

ILD
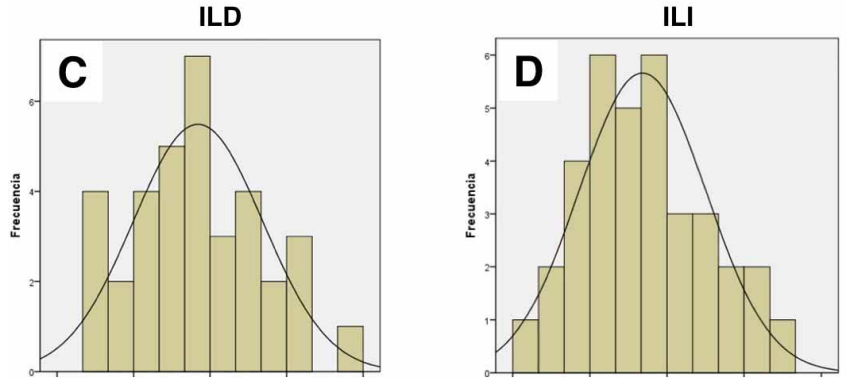

Fig. 2. Curvas de frecuencia de sujetos incluidos en la investigación, considerando incisivo central derecho (ICD), incisivo central izquierdo (ICI), incisivo lateral derecho (ILD), incisivo lateral izquierdo (ILI).

\section{DISCUSIÓN}

La angulación dentaria del incisivo maxilar normal ha sido analizada por algunos estudios, sin embargo, limitados análisis cefalómetros consideran este elemento; la posición del diente sobre sus base ósea es fundamental en la planificación y ejecución de tratamiento de pacientes con DF-III. Legan \& Burstone (1980) indicaron que la angulación observada normal era de $112^{\circ}$, aunque presentaron una desviación estándar de $5^{\circ}$, lo cual entregaría un variación importante que estaría en relación a la base ósea y a la cantidad de hueso disponible para esa posición. En esta área, estudios con tomografías computadorizadas son limitados y buena parte de estos análisis han sido realizadas con imágenes bidimensionales; en esas condiciones, es complejo definir las opciones de movilidad de dientes (con imagen 2D no se puede evaluar cantidad de hueso rodeando la raíz dentaria) y también es prácticamente imposible identificar las condiciones de incisivos laterales (que presentan menor cantidad de hueso al compararlo con incisivos centrales) (Kong et al., 2016).

Existe evidencia que demuestra las alteraciones y limitados resultados postoperatorios provocadas por la posición incorrecta del incisivo superior en términos de una mala posición en su base ósea y consecuentemente las dificultades que puede entregar una falta de descompensación pre quirúrgica en los protocolos convencionales de cirugía ortognática (Capelozza Filho et al., 1996); en protocolos de cirugía prime- ro, (Huang \& Chen) también han identificado estas valoraciones, entregando a la posición de incisivos un papel importante en la planificación quirúrgica.

Nuestros resultados muestran angulaciones de $113,7^{\circ}$ a nivel de incisivos centrales, próximos a la normalidad indicado por Legan \& Burstone; bajo esas angulaciones, sujetos sometidos a protocolo de cirugía primero podrían presentar buen rendimiento post quirúrgico; aunque es esperable que en condiciones de DF-III los incisivos maxilares se encuentren con una pro inclinación hacia anterior, aumentando la angulación en relación a la base palatina (Jazmati et al., 2016), nuestros resultados arrojan condiciones próximas a la angulación normal y a la relación adecuada con la base ósea ubicada, no obstante, en una posición retraída.

Tseng et al. (2011) realizaron un análisis para determinar las medidas cefalométricas que pueden relacionar la elección de tratamiento ortodoncico u orto-quirúrgico; de las 6 medidas seleccionadas como mas influyentes se observó que dos estaban relacionadas íntegramente con dientes, considerando un overjet menor de 4,73 $\mathrm{mm}$ y un overbite menor a $-0,18 \mathrm{~mm}$ como indicativos de cirugía; la inclinación de incisivos centrales van a influenciar fuertemente estas variables, de forma que la decisión terapéutica debe reconocer la angulación dentaria como un factor importante.

Pereira-Stabile et al. observaron angulaciones de $116,02^{\circ}$ en incisivos centrales en 50 sujetos con movimientos ortodoncicos previos a cirugía ortognática, demostrando que angulaciones mayores en sujetos con DFIII limitan movimientos maxilares, dificultando resultados funcionales y estéticos. En este sentido, Cao et al. (2011) señalaron que sujetos portadores de angulaciones mayores en incisivos centrales son, facialmente, menos atractivos bajo la observación de personas con y sin entrenamiento clínico.

Bajo conceptos de cirugía primero, la angulación de incisivos ha sido relacionada con posibilidad de recidivas de movimiento orto-quirúrgico por Ko et al. (2013), donde los grupos con mayor estabilidad de movimiento ortognático presentaron una angulación de incisivo central de $121,28^{\circ}\left( \pm 5,53^{\circ}\right)$ mientras que el grupo de menor estabilidad y mayor recidiva presentó angulaciones de $124,52^{\circ}$ $\left( \pm 6,93^{\circ}\right)$. Consideraciones de apiñamiento dentario, curva oclusal y proinclinación de incisivos son fundamentales en la elección de estrategias de cirugía primero (Yu et al., 2015), donde recomendaciones de protocolos ortodóncicos incluyen la posibilidad de $6^{\circ}$ de libertad para la ejecución de la ortodoncia post quirúrgica (Huang \& Chen). 
Se puede concluir que sujetos con DF-III presentan una angulación dentaria de incisivos superiores adecuadas para ejecutar procedimientos de cirugía ortognática bajo conceptos de cirugía primero.

AGRADECIMIENTOS. Los autores agradecen a la Dirección de Investigación de la Universidad de La Frontera por su financiamiento a través del proyecto UNT15-0008. Los autores Juan Pablo Alister Herdener y Francisca Uribe Fenner desean agradecer a La Comisión Nacional de Investigación Científica y Tecnológica, CONICYT, por financiar sus estudios de Doctorado.

BRITO, L.; VILLA, J.; NAVARRO, P.; ALISTER, J.P.; URIBE, F.; OLATE, S. Maxillary incisor inclination in subjects with class III facial deformity. Options in surgery first. Int. J. Morphol., 34(4):1502-1505, 2016

SUMMARY: The objective of this research was to determine the inclination of maxillary incisors in subjects with class III malocclusion. Were included 35 subjects with Class III facial deformity (DF-III) to analyze cone beam computed tomography; measurements were realized in the four maxillary incisors identifying the palatal plane considered between anterior and posterior nasal spine and dental axial axis from the union between coronal point and apical point; The results showed angulations from $111.6^{\circ}$ to $113.7^{\circ}$ for the incisors, which allows estimation of values close to normal dental angulation. It is concluded that the selected subjects present adequate dental angulation to perform surgery first.

KEY WORDS: Incisor inclination; Class III malocclusion; Surgery first.

\section{REFERENCIAS BIBLIOGRÁFICAS}

Capelozza Filho, L.; Martins, A.; Mazzotini, R. \& da Silva Filho, O. G. Effects of dental decompensation on the surgical treatment of mandibular prognathism. Int. J. Adult Orthodon. Orthognath. Surg., 11(2):165-80, 1996.

Cao, L.; Zhang, K.; Bai, D.; Jing, Y.; Tian, Y. \& Guo, Y. Effect of maxillary incisor labiolingual inclination and anteroposterior position on smiling profile esthetics. Angle Orthod., 81(1):121-9, 2011

Goulart, D. R.; Muñoz, P.; Olate, S.; de Moraes, M. \& Fariña, R. No differences in morphological characteristics between hyperplastic condyle and class III condyle. Int. J. Oral Maxillofac. Surg., 44(10):1281-6, 2015.

Kong, W. D.; Ke, J. Y.; Hu, X. Q.; Zhang, W.; Li, S. S. \& Feng, Y. Applications of cone-beam computed tomography to assess the effects of labial crown morphologies and collum angles on torque for maxillary anterior teeth. Am. J. Orthod. Dentofacial Orthop., 150(5):789-95, 2016.

Hernández-Alfaro, F. \& Guijarro-Martínez, R. On a definition of the appropriate timing for surgical intervention in orthognathic surgery. Int. J. Oral Maxillofac. Surg., 43(7):846-55, 2014.
Huang, C. S. \& Chen, Y. R. Orthodontic principles and guidelines for the surgery-first approach to orthognathic surgery. Int. J. Oral Maxillofac. Surg., 44(12):1457-62, 2015.

Jazmati, H. M.; Ajaj, M. A. \& Hajeer, M. Y. Assessment of facial soft tissue dimensions in adult patients with different sagittal skeletal classes using cone beam computed tomography. J. Contemp. Dent. Pract., 17(7):542$8,2016$.

Ko, E. W.; Lin, S. C.; Chen, Y. R. \& Huang, C. S. Skeletal and dental variables related to the stability of orthognathic surgery in skeletal Class III malocclusion with a surgery-first approach. J. Oral Maxillofac. Surg., 71(5):e215-23, 2013

Legan, H. L. \& Burstone, C. J. Soft tissue cephalometric analysis for orthognathic surgery. J. Oral Surg., 38(10):744-51, 1980.

Muñoz, G.; Olate, S.; Cantín, M.; Vásquez, B.; del Sol, M. \& Fariña, R. TMJ in facial class III deformity. Condyle/fossa relations. Int. J. Clin. Exp. Med, 7(9):2735-9, 2014a.

Muñoz, G.; Olate, S.; Cantín, M.; Vásquez, B. \& del Sol, M. TMJ in facial class III deformity. Condylar morphology relations. Int. J. Clin. Exp. Med, 7(9):3113-7, 2014b.

Olate, S.; Cantín, M.; Vásquez, B. \& Olate, G. Structure of the nose in patients with class III facial deformities. Int. J. Morphol., 32(1):334-8, 2014.

Olate, S.; Cantín, M.; Muñoz, M.; Alister, J. P.; Vásquez, B. \& Chaves Netto, H. Nasal deformity in subjects with class III facial deformities. Int. J. Morphol., 33(4):1536-41, 2015.

Pereira-Stabile, C. L.; Ochs, M. W.; de Moraes, M. \& Moreira, R. W. Preoperative incisor inclination in patients with Class III dentofacial deformities treated with orthognathic surgery. Br. J. Oral Maxillofac. Surg., 50(6):533-6, 2012.

Tseng, Y. C.; Pan, C. Y.; Chou, S. T.; Liao, C. Y.; Lai, S. T.; Chen, C. M.; Chang, H. P. \& Yang, Y. H. Treatment of adult Class III malocclusions with orthodontic therapy or orthognathic surgery: receiver operating characteristic analysis. Am. J. Orthod. Dentofacial Orthop., 139(5):e485-93, 2011.

Troy, B. A.; Shanker, S.; Fields, H. W.; Vig, K. \& Johnston, W. Comparison of incisor inclination in patients with Class III malocclusion treated with orthognathic surgery or orthodontic camouflage. Am. J. Orthod. Dentofacial Orthop., 135(2):146e1-9, 2009.

Yu, H. B.; Mao, L. X.; Wang, X. D.; Fang, B. \& Shen, S. G. The surgeryfirst approach in orthognathic surgery: a retrospective study of 50 cases. Int. J. Oral Maxillofac. Surg., 44(12):1463-7, 2015.

\section{Correspondence:}

Prof. Dr. Sergio Olate

Facultad de Odontología

Universidad de La Frontera

Claro Solar 115, oficina 414-A

CHILE

Email: Sergio.olate@ufrontera.cl

Recibido : 11-06-2016

Aceptado: 15-09-2016 